This is a preprint of an article accepted for publication in Color Research and Application () copyright 2004 by Wiley Periodicals Inc., a Wiley company.

\title{
Color Rendering: a Calculation That Estimates Colorimetric Shifts
} James A. Worthey, 11 Rye Court, Gaithersburg, Maryland 20878-1901, USA.

\begin{abstract}
Lights vary in their ability to render object spectral reflectances into color contrasts. When a light $L 1$ is replaced by another $L 2$, even if $L 2$ matches $L 1$ in chromaticity, systematic color shifts may occur, including a loss or gain of chromatic color. For instance, many familiar lights, when compared to daylight, dull red and green objects, rendering them closer to gray. An opponent colors method is appropriate to this discussion because it brings to the surface the notion of chromatic color, meaning actual departure from white or gray. In this article, an opponent-colors analysis leads to a matrix formulation that serves two purposes. The effects of replacing $L 1$ by $L 2$ are estimated with a $3 \times 3$ "rendering matrix" $\mathbf{P}$. Given an object's tristimulus vector under $L 1$, the method makes an approximate prediction of the new tristimulus vector under $L 2$. Thanks to the opponent formulation, matrix element $P 22$ quantifies the gain or loss of redness and greenness, while $P 33$ expresses gain or loss of blueness and yellowness. These in fact are major effects, so the method is both quantitative and explanatory.

Figures are in a separate file: http://www.jimworthey.com/RndrCalcFigs.pdf
\end{abstract}

Keywords: ASTM, Color Rendering, Colorimetry, Fluorescent Lighting, Illuminants, Lighting, Linear Model, Matrix R, Neodymium Glass, Opponent Color, Prime Colors, Standards.

\section{INTRODUCTION}

Lights vary in their ability to render object properties into black-white and color contrasts ${ }^{1}$. A previous article ${ }^{1}$ showed the systematic nature of color rendering effects that are likely to arise. Owing to the spectral overlap of red and green cone sensitivities, a light can be achromatic - stimulating all three cone systems - if it has power in only two narrow bands, one in the blue and one in the yellow. This makes a white light but renders reds and greens as black or brown. Familiar lights, such as Cool White fluorescent, when compared to a broad-band light such as daylight, dull reds and greens, taking a stride in the direction of the two-bands example. The need for actual red and green power in a light — not just a narrow band in the yellow-is expressed by Thornton's Prime Color analysis, by the diagonal of Cohen's Matrix $R$, or by an opponent-colors approach ${ }^{1,2}$.

The previous article ${ }^{1}$ is intended as an introduction to this one, which presents an explicit method. Jozef Cohen found that the smoothness of object reflectance spectra could be expressed by writing them as linear combinations of a few basis functions ${ }^{3}$. Extending that idea, he found that color matching functions themselves could be considered basis functions for any colored 
light, ${ }^{4}$ combining to give the "fundamental metamer," and leading on to Matrix $\mathrm{R}^{1,4}$. Sherman Lee Guth and others have created simple quantitative opponent colors models ${ }^{5}$. (See Appendix A.) In what follows, these ideas are merged (and slightly scrambled), using orthogonal function and matrix methods, distilling everything into a single equation with no adjustable parameters, Eq. (13). This formula gives an approximate prediction of object-color shifts under a change of lights, and in the process quantifies any overall effects on color contrasts. Prime color lights ${ }^{1,6}$ and other inventions provide revealing examples.

\section{Goal}

Suppose that a room contains objects of various colors. The lighting is changed from an initial light $L_{1}$ to a second light $L_{2}$ :

$$
L_{1} \rightarrow L_{2} \text {. }
$$

In general, all the objects change in tristimulus values. If there were only one colored surface, of spectral reflectance $s(\lambda)$, it would be a standard calculation to find the tristimulus values under $L_{1}$ and under $L_{2}$, and one could find the vector difference between the tristimulus vectors, everything calculated exactly. The vector difference is sometimes called the colorimetric shift. That is colorimetry, but it is not color rendering. A color-rendering method, in one way or another, should describe the overall effect of the lighting substitution.

The prototypical color rendering scenario is assumed to be a transition between two lights of the same chromaticity. To this extent, the problem at hand is the same one addressed by the CIE's CRI method ${ }^{13}$. However, this article treats color rendering as scientifically interesting, while the CRI method is based on hidden assumptions and tends to cut off discussion.

The method to be developed will model the colorimetric shifts of all objects under a lighting transition $L_{1} \rightarrow L_{2}$. In order to confront the issue of spectral overlap between red and green receptor sensitivities, surface tristimulus vectors will be expressed in an opponent color system. A square symmetrical matrix $\mathbf{P}$ will be derived such that if one knows a surface's tristimulus vector under $L_{1}, \mathbf{Q}\left(L_{1}\right)$, the matrix product $\mathbf{P Q}\left(L_{1}\right)$ will be a useful estimate of the tristimulus vector $\mathbf{Q}\left(L_{2}\right)$ after the lighting change. While any linear model of surfaces could lead to an illuminant matrix, the particular opponent formulation will allow the individual matrix elements to have intuitive meaning. For example, the element $P_{22}$ will be the gain for redness and greenness. In this way an orderly mathematical method will rediscover what was found empirically in the previous article: reds and greens are in greatest jeopardy from lights of poor color rendering.

\section{The Plan}

Let it not be said that I have buried my main ideas deep in the article. Omitting all nuance, here is the plan. Suppose the CIE $2^{\circ}$ observer sees a surface reflectance $s(\lambda)$ under a light $L_{1}(\lambda)$. The tristimulus values for surface $s(\lambda)$ are given by sums of the form $\Sigma \bar{x} L_{1} s$, where the sum is over $\lambda$. So far, no news. Recall that any 3 linear combinations of the members of $\{\bar{x}, \bar{y}, \bar{z}\}$ are a new set of color matching functions $\left\{\overline{q_{1}}, \overline{q_{2}}, \overline{q_{3}}\right\}$ that predict the same matches, so long as the transformation has determinant $\neq 0$. Now arrange such a set of color matching functions that are orthonormal, $\Sigma \bar{q}_{i} \bar{q}_{j}=\delta_{i j}$. Here $\delta_{i j}$ is the Kronecker delta, equal to one if $\mathrm{i}=\mathrm{j}$, and equal to zero otherwise; the sum is over $\lambda$. This is in fact easy to do, and if we start by making $\overline{q_{1}}=\overline{y /}\left(\Sigma \overline{y^{2}}\right)^{1 / 2}$, 
proportional to the usual luminance sensitivity $\bar{y}$, then the set of $\overline{q_{i}}$ 's can be made to look like opponent-color functions, though they will not be identical to any well-known opponent colors model. This set of color-matching functions is graphed in Fig. 1.

The orthonormal feature makes it easy to approximate other functions of wavelength by linear combinations of $\overline{q_{1}}, \overline{q_{2}}, \overline{q_{3}}$. At this point, let $\left|L_{1}\right\rangle$ be normalized thus:

$$
L_{1} \leftarrow L_{1}\left(\left\langle\overline{q_{1}} \mid \overline{q_{1}}\right\rangle /\left\langle\overline{q_{1}} \mid L_{1} \overline{q_{1}}\right\rangle\right)
$$

While Eq. (2) is somewhat arbitrary, a rationale will be given later. In this new color system, the tristimulus values, $Q_{i}$, can be written as

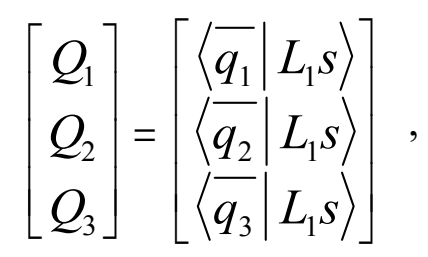

where the brackets, $\langle\mid\rangle$ indicate inner products - those sums over $\lambda$. Applying the orthonormal property, we can approximate $s$ by a truncated series:

$$
|s\rangle=\left|\overline{q_{1}}\right\rangle\left\langle\overline{q_{1}} \mid s\right\rangle+\left|\overline{q_{2}}\right\rangle\left\langle\overline{q_{2}} \mid s\right\rangle+\left|\overline{q_{3}}\right\rangle\left\langle\overline{q_{3}} \mid s\right\rangle+\ldots
$$

This leads to an approximate version of Eq. (3):

$$
\left[\begin{array}{l}
Q_{1} \\
Q_{2} \\
Q_{3}
\end{array}\right] \approx\left[\begin{array}{lll}
\left\langle\overline{q_{1}} \mid L_{1} \overline{q_{1}}\right\rangle & \left\langle\overline{q_{1}} \mid L_{1} \overline{q_{2}}\right\rangle & \left\langle\overline{q_{1}} \mid L_{1} \overline{q_{3}}\right\rangle \\
\overline{q_{2}}\left|L_{1} \overline{q_{1}}\right\rangle & \left\langle\overline{q_{2}} \mid L_{1} \overline{q_{2}}\right\rangle & \left\langle\overline{q_{2}} \mid L_{1} \overline{q_{3}}\right\rangle \\
\left\langle\overline{q_{3}} \mid L_{1} \overline{q_{1}}\right\rangle & \left\langle\overline{q_{3}} \mid L_{1} \overline{q_{2}}\right\rangle & \left\langle\overline{q_{3}} \mid L_{1} \overline{q_{3}}\right\rangle
\end{array}\right]\left[\begin{array}{l}
\left\langle\overline{q_{1}} \mid s\right\rangle \\
\left\langle\overline{q_{2}} \mid s\right\rangle \\
\left\langle\overline{q_{3}} \mid s\right\rangle
\end{array}\right] .
$$

If 3 inner products produce an exact answer in Eq. (3), why is it better to get an approximate answer using 12 inner products in Eq. (5)? Because Eq. (3) is colorimetry, but Eq. (5) is color rendering! The effects of illuminant $L_{1}$ are isolated in the square matrix, independent of the particular surface $s$.

The column matrix on the right summarizes the spectral reflectance $s$ by three numbers which are in fact the tristimulus values of $s$ under the equal-energy illuminant. The column matrix on the left contains the (approximate) tristimulus values of $s$ under $L_{1}$. Therefore, the square matrix is a transformation that takes as input the tristimulus values of any surface under the equalenergy light and estimates the new tristimulus values under $L_{1}$. In the introductory article ${ }^{1}$ were many little arrows within chromaticity diagrams, each representing a particular object color, first under one light, and then under another. Eq. (5) accepts any tristimulus vector as a starting point (arrow tail) and estimates the new tristimulus value (arrowhead). This is a powerful form of approximate prediction, similar to things done in the study of computer graphics and machine 
vision. (Yes, the results can be converted to $[X Y Z]^{\prime}$.)

Now consider what the tristimulus vector $\left[Q_{1} Q_{2} Q_{3}\right]^{\prime}$ means in an opponent-color system. The first element, $Q_{1}$, is the luminous reflectance of $|s\rangle$ under $\left|L_{1}\right\rangle$, or if $\left|L_{1}\right\rangle$ had not been normalized, $Q_{1}$ would be proportional to luminance. The second element, $Q_{2}$, represents redness or greenness of the lighted surface $|s\rangle . Q_{2}>0$ represents redness while $Q_{2}<0$ indicates greenness. Similarly, $Q_{3}>0$ denotes blueness, while $Q_{3}<0$ denotes yellowness. A white object, for instance, will have $Q_{1}$ equal to some nonzero number, such as 0.90 , but $Q_{2} \approx 0$ and $Q_{3} \approx 0$. To the extent that $Q_{2}$ and $Q_{3}$ differ from zero, they represent actual signals, a non-neutral chromaticity. Because $\left|L_{1}\right\rangle$ was normalized, Eq. (2), $Q_{2}$ and $Q_{3}$ are chromaticity coordinates, already adjusted for the intensity of the light.

\section{Meanings of matrix elements}

Since the tristimulus values represent signals, the elements of the square matrix are gains. The diagonal elements are the easiest to understand. The first diagonal element predicts luminous reflectance under $L_{1}$ as a multiple of luminous reflectance under the equal energy illuminant, $E$. The second diagonal element - the center element of the square matrix — predicts the redness or greenness of the surface as a multiple of its value under $E$. Recall that in the introductory article some lighting transitions systematically made reds redder and greens greener, or the reverse. This second diagonal element, if it differs from unity, predicts such a systematic effect in the transition from $E$ to $L_{1}$. The third diagonal element is the gain for blueness or yellowness.

For an example, suppose that $L_{1}=E$. That is to say, the test light $i s$ the equal energy light. Substituting the number 1.0 for $L_{1}$ in Eq. (5) and applying the orthonormality condition, $\left\langle\overline{q_{i}} / \overline{q_{j}}\right\rangle=$ $\delta_{i j}$, causes the square matrix to become an identity matrix. In other words, the tristimulus vector of $s$ under $E$ is equal to itself, a check on the method's logic.

Eq. (5) brings the color-rendering problem into the orderly realm of matrices and linear algebra. From this starting point, many steps can be taken. The transformation itself can be transformed, so that the tristimulus vectors refer to some other color system and not the orthonormalized one. A color-rendering matrix can be computed for any starting light $L_{1}$ and ending light $L_{2}$. As further inferences are drawn, it may begin to appear that whole method is extremely complicated. In fact, the central idea is contained in Eq. (5) and its interpretation. Further manipulations are just algebra. The ability to solve new problems should be seen as a benefit and not as "complication."

Eqs. (4) and (5) express a standard method in applied mathematics. The specialized features are these:

1. Object spectral reflectances are approximated by a linear combination of color-matching functions, rather than some other set of basis functions.

2. The color-matching functions are based on the CIE $2^{\circ}$ observer, but are linear combinations of $\{\bar{x}, \bar{y}, \bar{z}\}$ such that:

a. The cmf's are orthonormal, $\left\langle\overline{q_{i}} / \overline{q_{j}}\right\rangle=\delta_{i j}$.

b. The first $\mathrm{cmf}$ is essentially the familiar luminance sensitivity, $\langle\bar{y}|$, but normalized so that $\left\langle\overline{q_{1}} \mid \overline{q_{1}}\right\rangle=1.0$, in deference to orthonormality, item a. 
c. The second and third cmf's are opponent functions, making the 3 of them into an opponent set, similar to well-known opponent models.

3. The tristimulus values in the opponent scheme have more meaning than would $\{X, Y, Z\}$. In particular, $Q_{2}$ and $Q_{3}$ are "signals" that are small or zero for a neutral object, while one or both departs further from zero for a more saturated color.

4. The square matrix carries all the remaining information about $\left./ L_{1}\right\rangle$, including useful color-rendering information. Because it transforms one set of color signals into another, the 9 matrix elements can be thought of as gains. Each of those gains has an intuitive meaning, though the diagonal elements are the easiest to explain.

5. In particular, the second diagonal element is the direct gain for redness or greenness. It quantifies the quality of revealing or suppressing reds and greens, of which so much was said in the introductory article ${ }^{1}$.

6. In the plan as just stated, the matrix describes a lighting transition $L_{0} \rightarrow L_{1}$, where $L_{0}$ is the equal energy light. As the details unfold below, the method will be extended to allow any broad band light $L_{0}$ to be the initial light.

7. Though the CIE $2^{\circ}$ observer was used for concreteness, the same schema is easily applied with the $\mathrm{CIE} 10^{\circ}$ observer, or another standard observer that may be developed.

\section{Background: Smoothness Constraint}

Nature imposes a smoothness constraint on object colors: reflectances of most solids change slowly with wavelength. This can be expressed as a limit on the slope $\Delta s / \Delta \lambda$, where $s$ is spectral reflectance ${ }^{7}$, but a more popular method is to assume that most spectral reflectances can be expressed as linear combinations of a small set of basis functions ${ }^{3}$. That is to say, if $\left|s_{i}\right\rangle$ is the spectral reflectance of a particular object, then

$$
\left|s_{i}\right\rangle=\sum_{j=1}^{k} c_{i j}\left|b_{j}\right\rangle+\ldots,
$$

where $k$ is a fairly small number such as 3,4 , or 5 , and the basis functions $\left\{\left|b_{j}\right\rangle, j=1, k\right\}$ are a fixed set of linearly independent functions common to all objects under discussion, however numerous the objects. The notation "...$+ "$ means that the sum of terms only approximates $\left|s_{i}\right\rangle$, and the fit could be improved if $k$ were increased. Eq. (6) is sometimes said to express $\left|s_{i}\right\rangle$ by a linear model ${ }^{8,9,10}$.

Vrhel, Gershon and Iwan cite several theoretical articles that have depended on Eq. (6) with $k$ small $^{10}$. Their goal was to test this hypothesis by measuring 354 spectral reflectances, and performing a principal components analysis on the entire collection of spectral functions to derive the mean $\left|b_{0}\right\rangle$ and 7 basis functions $\left|b_{j}\right\rangle$. This implies a slightly different form of the linear model, shared by other famous principal components models ${ }^{11,12}$ :

$$
\left|s_{i}\right\rangle=\left|b_{0}\right\rangle+\sum_{j=1}^{k} c_{j}\left|b_{j}\right\rangle+\ldots
$$

For their data, Vrhel et al. found this approximation to be a mediocre one for $k=3$, but much 
better when $k=4$. When the fit is based on principal components, Eq. (7), the number of vectors used is $k+1$, or one more than the "number of basis functions" to which Vrhel et al. refer.

The CIE's color rendering document ${ }^{13}$ does not mention spectral smoothness of object reflectances or linear models or anything of the sort. Among the hidden assumptions of the calculation resides an implicit linear model, since the universe of objects is represented by 8 Munsell colors. If the Munsell colors are spectrally dependent as Cohen found ${ }^{3}$, then the implicit model has 3 basis vectors; otherwise it has at most 8 basis vectors.

In this article, I approximate object reflectances by a model in the form of Eq. (6), with $k=3$. This excludes object color metamerism from the discussion. Increasing $k$ would permit metameric object colors, with some plan needed to supply the additional basis functions. Appendix B explains the notation ${ }^{14}$ used to keep track of orthogonal functions and inner products $^{2,15,16}$.

\section{DETAILED THEORY WITH PIVOTAL LIGHT}

As a starting point I take the simple opponent-colors model explained in Appendix A. Within this model, receptor sensitivities are defined, $\{\langle\bar{r} \overline{\mid},\langle\bar{g}|,\langle\bar{b}|\}$, and then opponent-color sensitivities, $\{\langle\bar{a}|,\langle\bar{t}|,\langle\bar{d}|\}$. Each of these sets is a set of three color matching functions, equivalent to the CIE $2^{\circ}$ observer functions, $\{\langle\bar{x},\langle\bar{y}|,\langle\bar{z}|\}$, but with some additional meaning.

I now wish to approximate all object reflectances by linear combinations of 3 basis functions:

$$
\left|s_{i}\right\rangle=\sum_{j=1}^{3} c_{i j}\left|\bar{q}_{j}\right\rangle+\ldots
$$

This is Eq. (6) with $k=3$ and basis functions $\left|\overline{q_{\mathrm{j}}}\right\rangle$, which are created as follows:

1. Begin with the opponent sensitivities, $\{|\bar{a}\rangle,|\bar{t}\rangle,|\bar{d}\rangle\}$.

2. Choose a pivotal light source $L_{0}$. It can be one of the lights to be compared. Because of the way the pivotal light enters later steps, it is desirable that it be a relatively broad-band light, such as JMW daylight ${ }^{11}$, blackbody radiation, or the equal energy light E, not a peculiar source like low-pressure sodium.

3. Anticipating the next step, normalize $L_{0}$ so that it does not affect the normalization of $|\bar{a}\rangle$. That is, $L_{0} \leftarrow L_{0}\left(\langle\bar{a} \mid \bar{a}\rangle /\left\langle\bar{a} \mid L_{0} \bar{a}\right\rangle\right)$, Eq. (2).

4. The Gram-Schmidt process operates on functions in a specified sequence, and the first function is merely normalized, to become the first member of the orthonormalized set. The chosen sequence is $|\bar{a}\rangle,|\bar{t}\rangle,|\bar{d}\rangle$. Since $|\bar{a}\rangle$, called achromatic sensitivity, is the usual light-meter sensitivity $|\bar{y}\rangle$ times a constant, the first member of the orthonormal set will still be $|y\rangle$ times a constant. Now perform the Gram-Schmidt orthonormalization, but when an inner product is taken within the method, include $L_{0}$ as a weighting function.

5. The resulting orthonormal set may be called $\left\{\left|\overline{q_{1}}\right\rangle,\left|\overline{q_{2}}\right\rangle,\left|\overline{q_{3}}\right\rangle\right\}$.

Because the weighting function was used within the Gram-Schmidt process, the orthonormality 
condition is now

$$
\left\langle\overline{q_{i}} / L_{0} \overline{q_{j}}\right\rangle=\delta_{i j} .
$$

See Appendix C for a review of Gram-Schmidt orthonormalization as it applies here. Because a light $L_{0}$ multiplies a color matching function in Eq. (9), this method is similar to that of Keusen ${ }^{40}$.

Now Eq. (3) was the familiar formula for the tristimulus vector, and it still is:

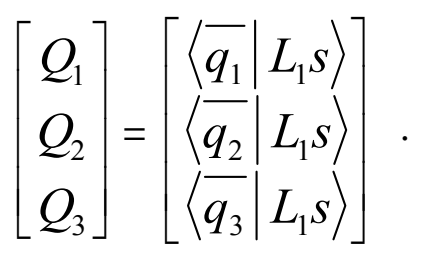

That is to say, the weighting factor $L_{0}(\lambda)$ does not appear spontaneously. In the summary of the plan, Eq. (5) was not explained. The more general version, with the weighting function, will now be derived. Let Eq. (8) be multiplied on the left by $\left\langle\overline{q_{m}} L_{0}\right|$ :

$$
\left\langle\bar{q}_{m} L_{0} \mid s_{i}\right\rangle=\sum_{j=1}^{3} c_{i j}\left\langle\bar{q}_{m} L_{0} \mid \bar{q}_{j}\right\rangle+\ldots .
$$

By the orthonormality condition (9), the inner product on the right is $\delta_{m j}$, and only the $m$ term in the sum is nonzero, so we find

$$
c_{i m}=\left\langle\overline{q_{m}} L_{0} \mid S_{i}\right\rangle .
$$

This is the formula for each of the coefficients $c_{i j}$ in Eq. (10). Considering the $r$ th row in Eq. (3), and combining that with (8) and (11),

$$
Q_{r}=\left\langle\bar{q}_{r} L_{1} \mid s_{i}\right\rangle=\sum_{j=1}^{3}\left\langle\bar{q}_{r} L_{1} \mid \bar{q}_{j}\right\rangle\left\langle\bar{q}_{j} L_{0} \mid s_{i}\right\rangle+\ldots .
$$

Returning to matrix notation,

$$
\left[\begin{array}{c}
Q_{1} \\
Q_{2} \\
Q_{3}
\end{array}\right]=\left[\begin{array}{lll}
\left\langle\overline{q_{1}} \mid L_{1} \overline{q_{1}}\right\rangle & \left\langle\overline{q_{1}} \mid L_{1} \overline{q_{2}}\right\rangle & \left\langle\overline{q_{1}} \mid L_{1} \overline{q_{3}}\right\rangle \\
\left\langle\overline{q_{2}} \mid L_{1} \overline{q_{1}}\right\rangle & \left\langle\overline{q_{2}} \mid L_{1} \overline{q_{2}}\right\rangle & \left\langle\overline{q_{2}} \mid L_{1} \overline{q_{3}}\right\rangle \\
\left\langle\overline{q_{3}} \mid L_{1} \overline{q_{1}}\right\rangle & \left\langle\overline{q_{3}} \mid L_{1} \overline{q_{2}}\right\rangle & \left\langle\overline{q_{3}} \mid L_{1} \overline{q_{3}}\right\rangle
\end{array}\right]\left[\begin{array}{c}
\left\langle\overline{q_{1}} L_{0} \mid s_{i}\right\rangle \\
\left\langle\overline{q_{2}} L_{0} \mid s_{i}\right\rangle \\
\left\langle\overline{q_{3}} L_{0} \mid s_{i}\right\rangle
\end{array}\right]+\ldots
$$

This is the central result, a more general version of Eq. (5). Suppose, for example, that $L_{0}=\mathrm{D} 65$, the standard light representing $6500 \mathrm{~K}$ daylight. Then the column matrix on the right contains the tristimulus values of $\left|s_{i}\right\rangle$ under D65. Three numbers serve as a proxy for the complete spectral reflectance function, and those numbers contain the same information that is routinely used in 
industrial color discussions. It is not in the usual format $[X Y Z]^{\prime}$, but can easily be converted to or from that form. The conversion would be exact, except for some tiny roundoff error.

Referring to Eq. (13), let the tristimulus vector on the left be called $Q\left(\perp, L_{1}, s_{i}\right.$, est), where $\perp$ stands for "orthonormal basis," and "est" stands for "estimated." Since 4 modifiers are unwieldy, we can omit any that can be inferred from context. The vector on the right is $Q\left(\perp, L_{0}, s_{i}\right)$. The square matrix can be called $\mathbf{P}\left(\perp, L_{0} \rightarrow L_{1}\right)$ :

$$
\mathbf{P}\left(\perp, L_{0} \rightarrow L_{1}\right)=\left[\begin{array}{ccc}
\left\langle\overline{q_{1}} \mid L_{1} \overline{q_{1}}\right\rangle & \left\langle\overline{q_{1}} \mid L_{1} \overline{q_{2}}\right\rangle & \left\langle\overline{q_{1}} \mid L_{1} \overline{q_{3}}\right\rangle \\
\left\langle\overline{q_{2}} \mid L_{1} \overline{q_{1}}\right\rangle & \left\langle\overline{q_{2}} \mid L_{1} \overline{q_{2}}\right\rangle & \left\langle\overline{q_{2}} \mid L_{1} \overline{q_{3}}\right\rangle \\
\left\langle\overline{q_{3}} \mid L_{1} \overline{q_{1}}\right\rangle & \left\langle\overline{q_{3}} \mid L_{1} \overline{q_{2}}\right\rangle & \left\langle\overline{q_{3}} \mid L_{1} \overline{q_{3}}\right\rangle
\end{array}\right] .
$$

That is, $\mathbf{P}$ is the color-rendering matrix. The boldface 'P' may be thought of as an uppercase Rho, for Rendering. In this notation,

$$
\mathbf{Q}\left(\perp, L_{1}, s_{i}, \text { est }\right)=\mathbf{P}\left(\perp, L_{0} \rightarrow L_{1}\right) \mathbf{Q}\left(\perp, L_{0}, s_{i}\right) .
$$

\section{Meanings of Matrix Elements}

If $L_{1}=L_{0}$, applying the orthonormality condition of Eq. (9) in Eq. (14) gives $\mathbf{P}=\mathbf{I}$, the identity matrix. To emphasize:

$$
\text { For do-nothing transition, } L_{0} \rightarrow L_{0}, \mathbf{P}=\left[\begin{array}{lll}
1 & 0 & 0 \\
0 & 1 & 0 \\
0 & 0 & 1
\end{array}\right] \text {. }
$$

As $L_{1} \neq L_{0}$ causes an element to depart from its do-nothing value of 1 or 0 , this expresses some systematic effect. Recall the meanings of the tristimulus vector $\left[Q_{1} Q_{2} Q_{3}\right]^{\prime}$ :

$Q_{1}=$ luminous reflectance, also known as $Y$, or lightness.

$Q_{2}=$ redness if positive, or greenness if negative.

$Q_{3}=$ blueness if positive, or yellowness if negative.

With this in mind, the 9 matrix elements carry old into new as follows:

$P_{11}=$ lightness into lightness

$P_{22}=$ red into red or green into green

$P_{33}=$ blue into blue or yellow into yellow

$P_{12}=$ red or green into lightness

$P_{13}=$ blue or yellow into lightness

$P_{21}=$ lightness into red or green

$P_{23}=$ blue or yellow into red or green

$P_{31}=$ lightness into blue or yellow

$P_{32}=$ red or green into blue or yellow 
Simplest to discuss are the diagonal elements. The first, $P_{11}$, represents mapping of lightness into lightness, and if $L_{0}$ and $L_{1}$ were not normalized, this value could be a measure of how the transition $L_{0} \rightarrow L_{1}$ affects lightness. If $L_{1}$ is in fact normalized as $L_{0}$ was, to hold $\left\langle\bar{a} \mid L_{1} \bar{a}\right\rangle=1$, then $P_{11}=1$. The next diagonal element, $P_{22}$, tells how $L_{0} \rightarrow L_{1}$ affects reds and greens. If $P_{22}<1$, reds and greens tend to be duller and less saturated, for instance. $P_{33}$ acts similarly on blues and yellows. The off-diagonal elements express the mapping of one hue into another as detailed above. For instance, $P_{21}$ represents a light's ability to translate lightness into redness.

Inspection of Eq. (14) shows that $\mathbf{P}$ is always symmetrical, so that $P_{21}=P_{12}$, etc. This symmetry implies that when $P_{21}<0$, for instance, light-colored objects become more greenish, and greenish objects become lighter. While all mathematical variables have precise meanings, the color names red, green, etc. are used loosely to refer to the opponent dimensions $Q_{2}$ and $Q_{3}$, which are not aligned with pure or unique hues.

\section{Detailed Examples}

Fig. 2 illustrates the working of the model for the transition from JMW daylight ${ }^{11}$ to Cool White fluorescent light. The lights have the same chromaticity, shown in the figure by + . Forcing this match puts the "daylight" out of the normal band ${ }^{11}$ by +0.0025 units in $x$, a tiny deviation. The 36 arrow tails are chromaticities for a subset of the Munsell reflectances measured by Vrhel et $a l .{ }^{10}$, and calculated in the daylight condition by the normal detailed formula. Likewise, the normal formula gives the heads of the solid arrows under Cool White. The heads of the dashed arrows are calculated from the arrow tails by the linear approximation of Eq. (13).

In this case, the rendering matrix is

$$
\mathbf{P}(\perp, \text { JMW Daylight } \rightarrow \text { Cool White })=\left[\begin{array}{ccc}
1 & 0.0881 & -0.0158 \\
0.0881 & 0.7565 & 0.0077 \\
-0.0158 & 0.0077 & 1.0103
\end{array}\right] .
$$

This is the transformation between tristimulus vectors in the orthonormal basis. For the purpose of creating Fig. 2, it was converted to a transformation for tristimulus vectors expressed as [ $\left.\begin{array}{lll}X & Y & Z\end{array}\right]^{\prime}$, as explained in Appendix D. Similar to examples in the introductory article, Fig. 2 shows reds losing redness and greens losing greenness in this lighting change. The linear approximation (dashed arrows) is more accurate for some papers than for others, but in this example it expresses well the overall loss of red and green.

This, of course, was the goal: to express in numbers the main effects of a light such as Cool White fluorescent. In $\mathbf{P}(\perp$, Daylight $\rightarrow$ Cool White), Eq. (17), the matrix element that differs most from its do-nothing value is $P_{22}=0.7565$, the gain for redness or greenness.

In Fig. 3, the lights and explanation are the same, except that the example colors are the 8 Munsell reflectances tabulated in the Color Rendering Index method ${ }^{13}$. Again, the linear approximation is reasonable in the chromaticity shifts that it predicts. The small set of desaturated colors scarcely displays the systematic effects seen when purer reds and greens are 
considered. The CRI method ${ }^{13}$ makes no reference to the systematic nature of color rendering. Even if the method were augmented to call for a graphical display akin to the solid arrows in Fig. 3 , the reliance on 8 pastel chips would obscure the facts.

In Fig. 4, one would assume that the two lights compared both offer good color rendering. The transition is from $10,000 \mathrm{~K}$ daylight, to a warmer daylight phase, $4800 \mathrm{~K}$. Again, solid arrows show the exact colorimetric shift, while dashed arrows are found from the linear approximation, specifically

$$
\mathbf{P}(\perp, \mathrm{JMW} 10,000 \rightarrow \mathrm{JMW} 4800)=\left[\begin{array}{ccc}
1 & 0.1333 & -0.0074 \\
0.1333 & 1.1080 & 0.0820 \\
-0.0074 & 0.0820 & 0.5277
\end{array}\right] .
$$

Math mavens will see that there is an implicit constancy model here. To the extent that a lighting shift can be modeled by a matrix, constancy can be achieved by inverting the matrix. This is not a new idea, but the linkage of the rendering and constancy discussions may be novel.

\section{Diverse collection of lights}

Eq. (13) quantifies the rendering of light $L_{1}$ by comparison to a starting light $L_{0}$. Light $L_{0}$ was called the pivotal light because, in some cases, one might wish to compare a set of lights $\left\{L_{1}, L_{2}\right.$, $\left.L_{3}, \ldots\right\}$, each in turn, to the same initial light $L_{0}$. In Figs. 5 and 6, numerous lights are compared to the Equal Energy Light $\left(L_{0}=1\right.$ for all $\lambda$ ). In both cases, the abscissa is $10^{6}$ (color temperature) as calculated for each light. The color rendering matrix $\mathbf{P}\left(\perp\right.$, Equal Energy $\left.\rightarrow L_{j}\right)$ was calculated for each light $L_{j}$. From each such matrix, $P_{22}$ is graphed in Fig. 5 and $P_{33}$ is graphed in Fig. 6.

Blackbody spectra were calculated for 22 temperatures, leading to the values plotted as solid curves. For JMW daylight ${ }^{11}$, there is a permitted band about a mean locus in $(x, y)$. The + symbols denote four daylights on the mean locus. (In Fig. 5, one + is lost under a $\mathbf{A}$.) The greenish limit is traced by diamonds, $\diamond$, and the purplish limit by circles, $\bigcirc$. Curves could be drawn to connect these daylight points, but they are left out to reduce clutter. The daylight points appear in brown. Color is used redundantly with some shapes, to help sets of related points stand out. The pivotal light, Equal Energy, gives a rendering matrix that is the $3 \times 3$ identity, meaning $P_{22}=P_{33}=1.0$; the correlated color temperature happens to be $5456 \mathrm{~K}$. These points are plotted with a filled square, $\mathbf{\square}$, and the designation EQEN.

The bold triangles, $\triangle$, 2BDS, are a 2-bands light matched in chromaticity to $4002 \mathrm{~K}$ blackbody. The colorimetric effects of this peculiar light were mapped in Fig. 1 of the prior article ${ }^{1}$. Based on logic and example calculations, it was asserted that this light would lose reds and greens. The matrix theory then should yield $P_{22}$ small or zero. The rendering matrix now is 


$$
\mathbf{P}(\perp, \text { Equal Energy } \rightarrow \text { 2-bands })=\left[\begin{array}{ccc}
1 & 0.3025 & -0.0155 \\
0.3025 & 0.0953 & -0.0558 \\
-0.0155 & -0.0558 & 0.6967
\end{array}\right],
$$

so that $P_{22} \approx 0.1$. This value is not zero only because the two narrow bands ( $446 \mathrm{~nm}, 574 \mathrm{~nm}$ ) do not align with the intrinsic blue-yellow direction in the particular color space. For confirmation, the determinant of this matrix is $\operatorname{det}(\mathbf{P})=9.576 \times 10^{-18}$, or essentially zero. This shows that the transformation cannot be inverted, consistent with all tristimulus vectors being collapsed into a plane.

Other symbols indicate man-made lights, except for the asterisks, $*$. Open triangles denote the most traditional vapor discharge lights:

$\Delta$, Seven classic fluorescent light spectra from Wyszecki and Stiles's book ${ }^{17}:$ STWM $=$ Standard Warm White; WHIT = White; DLGT = Daylight; WMDX = Warm White Deluxe; SOFT = Soft White; CWDX = Cool White Deluxe.

$\triangle$, Other traditional lights: HPMV $=$ High Pressure Mercury Vapor ${ }^{18} ; \mathrm{HAL}=$ Metal Halide $^{19}$ (a vapor discharge lamp not to be confused with tungsten-halogen); CLWT $=$ Cool White Fluorescent, recent measurement ${ }^{20} ;$ HPS $=$ High Pressure Sodium ${ }^{18}$.

Both thin and bold open squares denote lights for which special claims of clear seeing have been made:

$\square, \mathrm{V}=$ Vita-Lite ${ }^{21}$; LUCH $=$ LumiChrome $1 \mathrm{XX}^{22} ; \mathrm{PR} 30^{23}, \mathrm{PR}^{2} 1^{23}, \mathrm{PR}^{2} \mathrm{~K}^{19}, \mathrm{PR} \mathrm{K}^{19}$ Prime Color fluorescent tubes, as designed by Thornton, but no longer sold. $\mathbf{\square}$, S35, 1, S47 = Solux filtered tungsten-halogen types ${ }^{24}$.

Four asterisks denote idealized 3-band lamps, each adjusted to have the same chromaticity as one of the formerly commercial prime color fluorescent types:

*, Idealized prime color lights with all power in narrow bands at 450, 540, and $610 \mathrm{~nm}$.

Filled triangles denote commercially available lamps for which some color-rendering claim is made, $\boldsymbol{\Lambda}$ : PL30, PL35, PL41 = 3-band fluorescent tubes; 3, 2, MC4Q are metal halide types; MCNA is high-pressure sodium ${ }^{25}$.

Four filled diamonds, $\bullet$, show the example of a $2900 \mathrm{~K}$ blackbody source filtered by neodymium glass, as explained in the introductory article ${ }^{1}$. To recall, glass containing $\mathrm{Nd}_{2} \mathrm{O}_{3}$ has a narrow "notch" absorption band at $583 \mathrm{~nm}$, along with some absorption at other wavelengths. The absorption of the yellow light makes reds and greens more saturated. In Fig. 5, the diamonds, $\bullet$, are numbered $0,1.7,4.2$, and 6.3 , indicating the percentage of $\mathrm{Nd}_{2} \mathrm{O}_{3}$ in the glass, with a thickness of $1.2 \mathrm{~mm}$. In Fig. 6 , only the $6.3 \%$ point is labeled, but the $0 \%$ point is the one on the blackbody curve, and the others are in sequence.

The filled circle, $\boldsymbol{\bullet}, \mathbf{S}$, is the sulfur lamp. It is temporarily off the market, and the SPD is subject to change when it becomes available again ${ }^{26}$. 
The prototypical color-rendering comparison looks at two lights with the same chromaticity, but dissimilar spectra ${ }^{1}$. However, the matrix method applies for lights that are not a chromaticity match, Fig. 4. We see that blackbody radiation tends to reveal reds and greens better at lower temperature (Fig. 5), but blackbodies reveal blues and yellows better at higher temperatures (Fig. 6). The variation with temperature is greater for $P_{33}$ than for $P_{22}$. Curiously, $P_{22}$ and $P_{33}$ for mean JMW daylight (+ signs), as functions of correlated color temperature, track quite close to the curve for blackbody.

In Fig. 6, we see $P_{33}$ values bunching along the sloping curve for blackbody radiation, much more than do the $P_{22}$ values in the other figure. Most of the alphabetic labels have been omitted in Fig. 6 because they would pile up in an unreadable way. Every light, of course, has the same temperature and symbol in both figures.

\section{Insight}

Fluorescent lamps were introduced in 1938 or $1939^{27}$. Color rendering was discussed in the 1940s and the discussion became somewhat frozen in place with the initial publication of the Color Rendering Index in $1965^{13}$. The classic lamp types, $\Delta$, were a familiar reality for that earlier discussion; they shared a tendency to dull reds and greens, a flaw that was acknowledged and partially remedied by the "deluxe" designs. In general, the lights for which special claims have been made, $\square$, give higher values of $P_{22}$ than do the classic types at the same color temperatures. The idealized three-band lights, $*$, show that enhancement of reds and greens is also possible and three-phosphor lamps exploit this idea.

Blackbody is comparable to daylight in Figs. 5 and 6, within the domain of the JMW model, confirming blackbody as a reasonable standard of normality at lower temperatures. Nonetheless, low color temperatures give low values of $P_{33}$. Solux lamps, invented by Kevin McGuire ${ }^{28}$, are filtered tungsten-halogen sources designed to resemble daylight (or blackbody) at temperatures higher than the actual temperature of the filament. This bluer light gives higher $P_{33}$. An unfiltered tungsten-halogen source has a color temperature of $2900 \mathrm{~K}$ to $3000 \mathrm{~K}$. In Figs. 5 and 6 a filled diamond, $\diamond$, sits on the blackbody curve at $2900 \mathrm{~K}$. The Solux lamps, denoted by three bold squares, $\boldsymbol{\square}$, indeed fall near the curve of blackbodies, at higher $P_{33}$, the better for us to see blues and yellows.

The sequence of filled diamonds, $\diamond$, shows that a neodymium filter adjusts blackbody light in a different way, raising both $P_{22}$ and $P_{33}$, with little effect on correlated color temperature. Metal halide lamps, symbol HAL, a later invention than high pressure mercury vapor, HPMV, can be used in similar fixtures, but are less detrimental to reds and greens. Among the filled triangles, $\mathbf{\Lambda}, 3,2$ and MC4Q are newer metal halide types with still higher values of $P_{22}$. Vita-Lite, $\mathrm{V}$, was marketed as a full-spectrum lamp, said to resemble to daylight. Similar claims are or were made for LUCH, Lumichrome 1XX. As measured by $P_{22}$, these lights do indeed come closer to daylight than do many of the classic types.

In the case of PR7K and PR5K, we see that two of the Ultralume lights, $\square$, gave $P_{22}$ slightly higher than the matched "ideal" narrow-band lamp, *. This echoes a comment in the introductory article that "the 'prime colors' give a large triangular gamut, but not the largest 
possible." It appears that the green phosphor in these lights peaks to the gamut-increasing side of $540 \mathrm{~nm}$. To form her own opinion, the reader may refer to Fig. 7 and to Fig. 5 of the introductory article ${ }^{1}$. In Fig. 7, Ultralume $7000 \mathrm{~K}$ (solid curve) is compared to idealized prime color (3 narrow triangular bands) and JMW daylight of the same chromaticity (long dashes), as well as blackbody radiation of the same correlated color temperature (short dashes). The graphs are matched for luminous power, $Y$, except that the idealized prime color is scaled by a factor of 0.05 , in order to fit it on the same graph.

\section{Moment of Reflection}

Within Fig. 5 reside stories of evolving lamp designs. Warm White Deluxe, WMDX, was intended to have a similar color temperature to Standard Warm White, STWM, but to give better rendering of reds. The triangle for WMDX is above that for STWM, confirming that reds will be redder (and greens greener) under the "deluxe" light. Though WMDX falls short of the blackbody curve in its red-green gain $P_{22}$, PR30 scores well above it, the result of systematic engineering. Finally, an idealized prime color light plots as an asterisk slightly above the formerly commercial PR30. At higher color temperature, a similar sequence can be traced, from STCW to CWDX, then blackbody and average daylight, followed by PR41 and idealized prime color.

\section{DISCUSSION}

Because one narrow band in the yellow can stimulate two cone systems, a light comprising two narrow bands, one blue and one yellow, can match a given white chromaticity. Such a 2-bands light will not reveal red and green objects in their true colors. For the eye to distinguish red and green objects, light at actual red and green wavelengths must be supplied. This is clarified by the comparison to color television, where the phosphor chromaticities define a triangular gamut of possible mixtures ${ }^{1}$. Thornton's prime color analysis formalizes this idea and adds additional insight about the wavelengths that act strongly in color mixtures. Opponent color theory and Cohen's "Matrix R" analysis give similar results ${ }^{1}$.

Based on the notion of two independent hue dimensions, opponent theories lead to color matching functions that look like mathematically orthogonal functions. Usually they are not quite orthogonal, but I exploit this idea by deriving similar color-matching functions that are orthogonal.

Object reflectances tend to be spectrally smooth, which can be expressed by a linear model, Eq. (6). I let the basis functions of the linear model be the orthonormalized opponent-color functions, Fig. 1. This orthonormal set is not quite a fixed thing, since the functions are made orthogonal with a pivotal light as a weighting function, Eq. (9). From these assumptions - the usual "linear model" for spectral reflectances with a couple novel features - the matrix theory of color rendering is derived, Eq. (13).

The particular choice of basis functions and the resulting Eq. (13) have two benefits: 1. If the pivotal light is a popular source such as, say, D65, then the 3-vector representing the object color is equivalent to the usual tristimulus vector [ $\left.\begin{array}{lll}X & Y & Z\end{array}\right]^{\prime}$ for that object under D65, as seen by the $\mathrm{CIE} 2^{\circ}$ or $10^{\circ}$ observer, as desired. 
2. The illuminant matrix can be used to estimate new tristimulus values from old when $L_{1} \rightarrow L_{2}$, as in Fig. 2, but any linear model would do this. The opponent-color basis functions cause the elements of the illuminant matrix $\mathbf{P}$ to have simple meanings. Of particular interest are $P_{22}$, the gain for redness or greenness, and $P_{33}$, the gain for blueness or yellowness.

This simple meaning for $P_{22}$ leads us back to the starting point, the observation that many artificial lights seen in daily life attenuate reds and greens, relative to daylight or blackbody light. In Figs. 5 and 6, a large collection of natural and artificial lights are compared in two messy graphs. Inventions claiming to enhance color vision generally do so by increasing $P_{22}$ relative to some prior technology. An older invention, the neodymium filter, raises both $P_{22}$ and $P_{33}$ when used with a $2900 \mathrm{~K}$ blackbody source.

\section{Fundamental Metamers}

Other sets of basis functions could have been used, such as those derived from reflectance data by Vrhel et al $^{10}$. Also, an alternate algebraic method could find the illuminant matrix without the intermediate step of orthonormalizing the functions. In defense of the chosen method, I may say that it does work: in Figs. 2-4, it gives reasonable estimates to the colorimetric shifts of real objects, at least in the $(x, y)$ projection. The Gram-Schmidt procedure is more than idle algebra, since it is the vehicle by which the pivotal light enters the calculation. (In Eq. (14), $L_{0}$ is not seen in the square matrix, but it is implicit through the calculation of functions $\left|\overline{q_{j}}\right\rangle$.)

The linear model, Eqs. (8) and (11), represents a spectral reflectance as a combination of color matching functions. This is what Cohen called the fundamental metamer of the object ${ }^{4}$. If $L_{0}$ is the equal-energy light, the model is exactly Cohen's fundamental metamer, otherwise it is a variation on the same idea. One consequence is that the model gives the exact tristimulus vectors of objects under $L_{0}$. In Figs. 2-4, the dashed arrows can be thought of as coming entirely from the linear model, the arrow tails as well as the heads. By contrast, if a different set of basis functions were used, there would be an "error of the approximation" under $L_{0}$ (tail of dashed arrow) as well as under $L_{1}$. The linear model is in general not a good fit to the actual reflectance, but it embodies that aspect of the reflectance that is accessible to the standard observer under $L_{0}$.

\section{Reproducibility}

The method of Eq. (13) is clearly stated and deterministic. My software starts with the CIE $2^{\circ}$ color matching functions as supplied at $1 \mathrm{~nm}$ steps and 7 significant digits ${ }^{37}$. Appendices A and $\mathrm{C}$ describe the brief manipulations to create the orthonormal basis. The illuminant matrix $\mathbf{P}$, Eq. (14), is self-explanatory and computed by matrix multiplications in as little as one line of computer code; see Appendix D. Easily duplicated light source spectra include blackbody and JMW daylight.

One issue must be handled carefully in order that numerical results be completely reproducible. That is the matter of normalizing the light. An illuminant matrix $\mathbf{P}$ describes a substitution $L_{0} \rightarrow L_{1}$ and the mathematical logic could readily express the fact that $L_{1}$ has higher or lower illuminance than $L_{0}$. We want instead that $L_{1}$ 's absolute power level not affect the results. Assume first that the pivotal light is Equal Energy, $L_{0}=1$. Note that the first basis function $\left|\overline{q_{1}}\right\rangle$ is 
nearly unchanged by the Gram-Schmidt process; it is the familiar $|\bar{y}\rangle$, renormalized so that $\left\langle\overline{q_{1}} \mid \overline{q_{1}}\right\rangle=1$. To make $\left|\overline{q_{1}}\right\rangle$ invariant for other choices of pivotal light, $L_{0}$ is normalized so that it does not affect this equality, $\left\langle\overline{q_{1}} \mid L_{0} \overline{q_{1}}\right\rangle=1$, with $\left|\overline{q_{1}}\right\rangle$ not adjusted. This is taken as the canonical normalization, and $L_{1}$ is similarly normalized:

$$
L_{1} \leftarrow L_{1}\left(\left\langle\overline{q_{1}} \mid \overline{q_{1}}\right\rangle /\left\langle\overline{q_{1}} \mid L_{1} \overline{q_{1}}\right\rangle\right) .
$$

With attention to this detail, applying Eq. (2) to $L_{0}$ and to $L_{1}$, numerical results should be fully repeatable. When this is done, $P_{11}=1$, as in Eq. (17), for example.

\section{Cautionary remarks}

Please keep in mind that the method presented is inherently a matrix theory. In Figs. 5 and 6, the equal energy light is taken as the pivotal light and then for each light $L_{j}$, a rendering matrix $\mathbf{P}\left(\perp, L_{0} \rightarrow L_{j}\right)$ is computed, Eq. (14). Then two diagonal elements of that matrix are graphed, with color temperature on the horizontal axis. The figures are interesting and based on logic. However, it would be wrong to begin taking ratios among values of $P_{22}$, say, and make inferences from that. What is authorized by logic is to use the operations of matrix inverse and matrix multiplication to estimate something. For instance,

$\mathbf{P}\left(\perp, L_{\mathrm{j}} \rightarrow L_{\mathrm{k}}\right) \approx \mathbf{P}\left(\perp, L_{0} \rightarrow L_{\mathrm{k}}\right) \mathbf{P}^{-1}\left(\perp, L_{0} \rightarrow L_{\mathrm{j}}\right)$. How accurate and useful this estimate might be, that is a question for another day.

This article is intended to discuss the general issue of color rendering, the derivation of a method, and the application of that method to some light sources of practical interest. The results are not meant as a consumer guide for comparing specific products. Some data were received directly from metrologists, while others may have been redrawn, with some loss of precision. In Fig. 5, two cool white fluorescent lights appear, STCW and CLWT, fairly dissimilar in the results they give. I consider the underlying spectra reliable in both cases. Therefore, there is some variation between vendors, or variation during the three decades that separate the measurements. To compare specific lights, the matrix method should be used with fresh test data.

\section{Object Colors in Real Life}

Color-meaning chromatic color and not just black-white - is part of normal vision and of normal life. Red-green contrast participates in edge detection ${ }^{29,30}$, and color can aid in segmenting the image. These basic steps precede recognition. Because of the eye's longitudinal chromatic aberration, color vision can and does participate in vergence accommodation - the focusing of the eye ${ }^{31}$. On a metallic object, say a brass teapot, highlights are the color of the metal, while a dielectric object, such as a ceramic teapot, has highlights that are white - approximately the color of the light source. Consider a red bell pepper on a gray background; color defines the vegetable's shape against the background, it identifies it as red, not green, and it may participate in the consumer's perception of quality. In focusing the eye, seeing the teapot or pepper, color rendering plays a role. To see the red or the green pepper properly, one needs red and green light. In a school, library, office or kitchen, full use of color vision requires actual red, green, and blue light, just as it does with a video screen. 
In this article and the preceding one ${ }^{1}$, we have seen that color rendering is a matter of systematic object chromaticity shifts. If broadband lights such as daylight and blackbody are the norm, then artificial lights often lose the chroma of reds and greens; similar effects on blues and yellows can also arise. This has been understood by a few in the lighting industry and elsewhere, but not clearly discussed or quantified. A number of inventors have understood and taken action to improve color rendering.

When a light systematically loses redness and greenness of objects, this is stimulus deprivation. The eye presumably adapts to the chromaticity of the source. After adaptation, what is needed for stimulation is that areas near to each other on the retina be far from each other in color space, in the dimensions of red vs green, blue vs yellow, and black versus white.

Broad statements are often made that "of course, daylight provides the best vision because humans evolved in daylight." Such remarks lead to a more technical question: what does daylight do that other lights fail to do? One answer is that the daylight spectrum affords good color contrasts, Figs. $5 \& 6$. A further answer is that the sun has high luminance and small angular subtense. (The sun covers $6 \times 10^{-5}$ sr or about $10^{-5}$ of the sky dome's $2 \pi$ sr.) Daylight thus engenders bright highlights, strong shading and cast shadows. Large area sources lose these stimuli and transform highlights - a source of shape information - into veiling reflections, which degrade the colors and contrasts of surfaces ${ }^{32}$. The effects of light source size are easier to describe in words and equations ${ }^{33}$ than are color rendering effects. However, lighting discussions often operate on the peculiar assumption that what is most obvious need not be studied. My point here is that many lights cause stimulus deprivation because of color rendering, but this is not the only way that lighting may degrade stimuli. Heschong-Mahone Group performed two studies showing clear benefits of daylight entering classrooms and retail stores by windows and skylights $^{34}$. In explaining the results within the school lighting report, the authors refer to highlights and color rendering as areas in which daylight may be superior to artificial lighting. My view is that such differences among lighting systems indeed exist ${ }^{33}$, and more can be done to discuss them in numbers and clear words.

\section{CONCLUSION}

Color rendering is not an issue of non-linearities in the visual system or elsewhere. The issues are basic and reside in the physical interaction of the light, the colored object, and the eye. If the eye were replaced by a television camera whose 3 sensitivity functions were not equivalent to human cone sensitivities, a similar matrix method could be derived for the camera. Under a 2bands light, the gamut of colors would collapse for the camera as for the eye.

\section{ACKNOWLEDGMENTS}

ASTM Committee E12 on Color and Appearance seeks to develop a new method to evaluate color rendering by lights. This article is intended as research in support of that goal, but is not a standard, nor approved by ASTM International, formerly the American Society for Testing and Materials. William Thornton, Nick Lena, and Philip Bradfield contributed data. Nicholas Worthey helped with manipulation of some input data. Calculations and graphs were done with a software package called O-Matrix. Reader comments are welcomed, by mail, by email to 
jim@jimworthey.com, or by phone to 1-301-977-3551 in the USA.

\section{Appendix A: An opponent-colors model similar to Guth's}

In past articles ${ }^{2,15,16}$, I have used an opponent-colors model that was developed by Sherman Lee $\mathrm{Guth}^{5}$. He developed this model to describe such phenomena as adaptation at a stage beyond the visual receptors. Looking back at what I did with this model, it was useful for its simplicity, and not so much for its intended meaning.

Guth's original $\operatorname{model}^{5}$ depended on receptor sensitivities ${ }^{36}$ that in turn depended on a slightly modified version of the CIE $2^{\circ}$ color-matching functions. For simplicity, I now use Guth's transformations, but apply them to the 1-nm version of the unmodified CIE functions ${ }^{17,37}$. Where the original model had weighting factors at the receptor and opponent levels ${ }^{2,5}$, I set those to unity, which allows them to be omitted. The receptor sensitivities are then expressed in terms of the workaday CIE color matching functions by:

$$
[\langle\bar{r}|\langle\bar{g}|\langle\bar{b}|]^{\prime}=\mathbf{M}_{1}[\langle\bar{x}|\langle\bar{y}|\langle\bar{z}|]^{\prime}
$$

where $\quad \mathbf{M}_{1}=\left[\begin{array}{ccc}0.2435 & 0.8524 & -0.0516 \\ -0.3954 & 1.1642 & 0.0837 \\ 0 & 0 & 0.6225\end{array}\right]$,

and prime, ', indicates matrix transpose. (Only the indicated $1 \times 3$ row matrices are transposed. The bras become the rows.)

The receptor outputs are added and subtracted to give opponent-color signals:

$$
[\langle\bar{a}|\langle\bar{t}|\langle\bar{d}|]^{\prime}=\mathbf{M}_{2}\left[\left\langle\bar{r} \overline{\mid}\langle\bar{g}|\langle\bar{b}]^{\prime}\right.\right.
$$

where $\quad \mathbf{M}_{2}=\left[\begin{array}{ccc}0.5967 & 0.3654 & 0 \\ 0.9553 & -1.2836 & 0 \\ -0.0248 & 0 & 0.0483\end{array}\right]$.

While it is interesting to see the receptor sensitivities, the opponent-color result is of primary interest. To go from the familiar color matching functions to opponent ones in one step, we notice that

$$
[\langle\bar{a}|\langle\bar{t}|\langle\bar{d}|]^{\prime}=\mathbf{M}_{2} \mathbf{M}_{1}[\langle\bar{x}|\langle\bar{y}|\langle\bar{z}|]^{\prime},
$$


where

$$
\mathbf{M}_{2} \mathbf{M}_{1}=\left[\begin{array}{ccc}
0 & 0.9341 & 0 \\
0.7401 & -0.6801 & -0.1567 \\
-0.0061 & -0.0212 & 0.0314
\end{array}\right] \text {. }
$$

A technicality intrudes here. Eq. (A6) is the "official" version of these numbers from reference 5. This is what Guth intended, particularly that the achromatic sensitivity $\langle\bar{a}|$ be equal to the familiar $\langle\bar{y}|$ times the constant 0.9341. When I multiply the matrices $\mathbf{M}_{2}$ and $\mathbf{M}_{1}$ using double or single precision arithmetic, I get discrepancies in the fourth decimal place such that, for instance, the two zeroes are not zero. In some actual calculations, I may indeed use my calculated value for $\mathbf{M}_{2} \mathbf{M}_{1}$, rather than Eq. (A6).

The small numerical discrepancies are not important. Quite important is Guth's reading on the meaning of the traditional CIE luminosity function $=V_{\lambda}=\langle\bar{y}$. His research had focused on heterochromatic brightness additivity, meaning experiments in which a subject is presented with two fields of different colors, and reports which is brighter, or adjusts one field to make them equally bright. In those experiments, colors add vectorially, not as predicted by the scalar summation built into the usual light meter. This is not construed to mean that "light meters are wrong," but rather that traditional photometry predicts the response of one of the three secondstage systems within the visual system. Heterochromatic brightness experiments elicit a response that can be described in terms of vector addition of the second-stage responses, $[\langle\bar{a} \mid S\rangle$ $\langle\bar{t} \mid S\rangle\langle\bar{d} \mid S\rangle]^{\prime}$, where $|S\rangle$ is the stimulus light ${ }^{38}$.

The key idea is this: traditional photometry, embodied in $\langle\bar{y}|$ and equally embodied in $\langle\bar{a}|$, is valid but is not the whole story. What is the rest of the story? It is everything to do with color, including vector brightness and color matching. Color rendering, as treated in this article, is based on the facts of color matching.

Now, what are the 3 functions $\langle\bar{a}|,\langle\bar{t}|,\langle\bar{d}|$ ? First of all, they are color-matching functions, completely equivalent to $\langle\bar{x},\langle\bar{y}|,\langle\bar{z}|$. Further, $\langle\bar{a}|=0.9341\langle\bar{y}|$, in other words $\langle\bar{a}|$ is the usual luminance sensitivity function, multiplied by a scalar constant. Function $\langle\bar{t}|$ is red-green sensitivity, called the tritanopic function by Guth because it is the subsystem that even a tritanope, a blue-blind person, would have. Function $\langle\bar{d}|$ is blue-yellow sensitivity, called deuteranopic because it is the subsystem that even a deuteranope would have. The set of functions $\{\langle\bar{a}|,\langle\bar{t}|,\langle\bar{d}|\}$, when graphed, look as if they could be orthogonal functions. They are in fact not orthogonal, but are close enough that when they have been orthogonalized by the GramSchmidt procedure, Fig. 1, they still look like opponent-color functions. The function sets $\left\{\langle\bar{r} \overline{\mid},\langle\bar{g}|,\langle\bar{b}|\}\right.$ and $\{\langle\bar{a}|,\langle\bar{t}|,\langle\bar{d}|\}$, derived from the normal CIE $2^{\circ}$ functions by Eqs. (A1, A5), are graphed and discussed in the introductory article ${ }^{1}$. 


\section{Appendix B, Notation of Bras and Kets}

Much of the algebra is developed using "bras" and "kets"14,15, in order to make visible the transition between orthogonal function methods, and matrix algebra. An alternate approach treats all functions as matrices from the beginning ${ }^{39}$. Functions of wavelength, say $f(\lambda), g(\lambda)$, are represented by bras, $\langle f|$, or kets, $|g\rangle$. When a bra directly precedes a ket, the resulting bracket indicates an inner product:

$$
\begin{gathered}
\langle f \mid g\rangle=\int_{0}^{\infty} f(\lambda) g(\lambda) d \lambda, \text { or } \\
\langle f \mid g\rangle=\sum_{\lambda=360}^{830} f_{\lambda} g_{\lambda},
\end{gathered}
$$

for example.

A ket preceding a bra, $|g\rangle\langle f|$, does not indicate an inner product. If we let kets be column vectors, and let bras be row vectors, then $\langle f \mid g\rangle$ is a matrix product with a scalar result, consistent with Eq. (B2). In other words, $\langle f|$ is the transpose of $|f\rangle$. In $\langle f \mid g\rangle$, the vertical line is a sort of punctuation with little meaning. If a third function is inserted, as in $\langle f \mid L g\rangle$, it is understood that $L$ and $g$ are multiplied wavelength-by-wavelength.

This notation is intended to keep the concepts of inner products and orthogonal functions in view. In Eq. (15), the key result, every matrix element is seen to result from an inner product involving such meaningful functions as the light's SPD, the surface's reflectance, and the opponent basis functions.

\section{Appendix C: Gram-Schmidt Orthogonalization}

A review of the Gram-Schmidt procedure may help to make the overall discussion more clear. When derived with no weighting function, the orthonormal functions have a certain scale on the ordinate of Fig. (1). To minimize the effect of the weighting function $L_{0}(\lambda)$ on ordinate values, we apply the canonical normalization of Eq. (2):

$$
L_{0} \leftarrow L_{0}\left(\langle\overline{a \mid} \bar{a}\rangle /\left\langle\bar{a} \mid L_{0} \bar{a}\right\rangle\right)
$$

Eq. (C1) is an extra step preceding the usual G-S process. The arrow, $\leftarrow$, means that $L_{0}$ is replaced by something else, in this case by a re-scaled version of itself. This notation, used to avoid defining more and more variables, corresponds to what one often does in a computer program.

The initial functions, in sequence, are $\{\langle\overline{a \mid},\langle\bar{t}|\langle\bar{d}|\}$. The first function $\langle\bar{a}|$ is normalized to become $\left\langle\overline{q_{1}}\right|$ :

$$
\left\langle\overline{q_{1}}\right|=\langle\bar{a}| /\left(\left\langle\bar{a} \mid L_{0} \bar{a}\right\rangle\right)^{1 / 2}
$$

Then $\left\langle\overline{q_{2}}\right|$ is the second function, minus the projection of $\left\langle\bar{t}\right.$ onto $\left\langle\overline{q_{1}}\right|$ : 


$$
\left\langle\overline{q_{2}}\right|=\langle\bar{t}|-\left\langle\bar{t} \mid L_{0} \overline{q_{1}}\right\rangle\left\langle\overline{q_{1}}\right|
$$

Step (C3) is the key step, which makes $\left\langle\overline{q_{2}}\right|$ orthogonal to $\left\langle\overline{q_{1}}\right|$. Now normalize $\left\langle\overline{q_{2}}\right|$,

$$
\left\langle\overline{q_{2}}\right| \leftarrow\left\langle\overline{q_{2}}\right| /\left(\left\langle\overline{q_{2}} \mid L_{0} \overline{q_{2}}\right\rangle\right)^{1 / 2} .
$$

With orthonormal functions $\left\langle\overline{q_{1}}\right|$ and $\left\langle\overline{q_{2}}\right|$ determined, $\left\langle\overline{q_{3}}\right|$ is $\langle\overline{d \mid}$ minus the projection of $\langle\overline{d \mid}$ onto each of those:

$$
\left\langle\overline{q_{3}}\right|=\langle\bar{d}|-\left\langle\bar{d} \mid L_{0} \overline{q_{1}}\right\rangle\left\langle\overline{q_{1}}\right|-\left\langle\bar{d} \mid L_{0} \overline{q_{2}}\right\rangle\left\langle\overline{q_{2}}\right|
$$

Then $\left\langle\overline{q_{3}}\right|$ is normalized:

$$
\left\langle\overline{q_{3}}\right| \leftarrow\left\langle\overline{q_{3}}\right| /\left(\left\langle\overline{q_{3}} \mid L_{0} \overline{q_{3}}\right\rangle\right)^{1 / 2} .
$$

Now complete for three basis vectors, the process can be extended indefinitely. From each of the initial set of vectors is subtracted its projections onto all the previous orthonormal vectors. That result is then normalized and the process repeated.

For a quick review of what motivates this process, let Eq. (C3) be post-multiplied by $\left|L_{0} \overline{q_{1}}\right\rangle$ :

$$
\left\langle\overline{q_{2}} \mid L_{0} \overline{q_{1}}\right\rangle=\left\langle\overline{t \mid} L_{0} \overline{q_{1}}\right\rangle-\left\langle\bar{t} \mid L_{0} \overline{q_{1}}\right\rangle\left\langle\overline{q_{1}} \mid L_{0} \overline{q_{1}}\right\rangle \text {. }
$$

Since $\left\langle\overline{q_{1}} \mid L_{0} \overline{q_{1}}\right\rangle=1$, then $\left\langle\overline{q_{2}} \mid L_{0} \overline{q_{1}}\right\rangle=0$, which was the goal. Step (C3) causes $\left\langle\overline{q_{2}}\right|$ to be orthogonal to $\left\langle\overline{q_{1}}\right|$, step (C5) makes $\left\langle\overline{q_{3}}\right|$ orthogonal to $\left\langle\overline{q_{1}}\right|$ and to $\left\langle\overline{q_{2}}\right|$, and so forth.

\section{Appendix D: Fun with Orthonormal Functions}

Versions of Eq. (6) appear frequently in science and engineering:

$$
\left|s_{i}\right\rangle=\sum_{j=1}^{k} c_{i j}\left|b_{j}\right\rangle+\ldots
$$

To make this useful, two things are needed: a set of basis functions $\left|b_{j}\right\rangle$ appropriate to the task at hand, and a formula for the coefficients $c_{i j}$.

As for the set of color matching functions $\left|\overline{q_{\mathrm{j}}}\right\rangle$, used as basis functions in this article, they are at least nonzero over the proper domain - the visible spectrum. Regarding coefficients $c_{i j}$, the answer is simple. So long as the basis functions are orthonormal, the formula for the coefficients is always found by the same short derivation. This leads to a shorthand method for working with Eq. (6).

Suppose that functions $\left|b_{j}\right\rangle$ are orthonormal when inner products are taken with weighting function $L_{0}$, that is $\left\langle b_{i} L_{0} \mid b_{j}\right\rangle=\delta_{i j}$. Then multiply Eq. (6) on the left by $\left\langle b_{m} L_{0}\right|$ to give 


$$
\left\langle b_{m} L_{0} \mid s_{i}\right\rangle=\sum_{j=1}^{k} c_{i j}\left\langle b_{m} L_{0} \mid b_{j}\right\rangle+\ldots
$$

Applying the orthonormal property,

$$
\left\langle b_{m} L_{0} \mid s_{i}\right\rangle=\sum_{j=1}^{k} c_{i j} \delta_{m j}+\ldots
$$

In the sum, only when $j=m$ is $\delta_{m j}=1$, rather than 0 . Therefore, the coefficient is

$$
c_{i m}=\left\langle b_{m} L_{0} \mid s_{i}\right\rangle,
$$

and substituting Eq. (D3) into Eq. (6) yields

$$
\left|s_{i}\right\rangle=\sum_{j=1}^{k}\left|b_{j}\right\rangle\left\langle b_{j} L_{0} \mid s_{i}\right\rangle+\ldots
$$

The bracket on the right is the explicit expression for coefficient $c_{i j}$. A physicist would say that in Eq. (D4), $\left|s_{i}\right\rangle$ is expanded in the basis $\left|b_{j}\right\rangle$, though he would prefer to have an infinite set of basis functions. Instead of using "..+ " to denote additional terms, we can write

$$
\left|s_{i}\right\rangle \approx \sum_{j=1}^{k}\left|b_{j}\right\rangle\left\langle b_{j} L_{0} \mid s_{i}\right\rangle
$$

agreeing that this has the same meaning as Eq. (D4).

Inspecting Eq. (D5), we see $\left|s_{i}\right\rangle$ on both sides, but on the right-hand side, it has additional symbols in front of it. Since these symbols operate on $\left|s_{i}\right\rangle$ and return the same vector, they act as a unity operator ${ }^{35}$. Using the symbol $\mathbb{1}$ for the unity operator,

$$
\begin{gathered}
\mathbb{1} \approx \sum_{j=1}^{k}\left|b_{j}\right\rangle\left\langle b_{j} L_{0}\right| \text { to expand a ket, }|f\rangle \text { or } \\
\mathbb{1} \approx \sum_{j=1}^{k}\left|L_{0} b_{j}\right\rangle\left\langle b_{j}\right| \text { to expand a bra, }\langle f| \text {. }
\end{gathered}
$$

Absent the weighting function $L_{0}$, the two forms would be the same. The unity operator allows Eq. (12) to be derived in one step:

$$
Q_{r}=\left\langle\bar{q}_{r} L_{1} \mid s_{i}\right\rangle=\sum_{j=1}^{3}\left\langle\bar{q}_{r} L_{1} \mid \bar{q}_{j}\right\rangle\left\langle\bar{q}_{j} L_{0} \mid s_{i}\right\rangle+\ldots .
$$




$$
\mathbb{1} \approx \sum_{j=1}^{3}\left|\bar{q}_{j}\right\rangle\left\langle\bar{q}_{j} L_{0}\right|
$$

In other words, to expand the function $\left|s_{i}\right\rangle$ in terms of the function $\left|\overline{q_{\mathrm{j}}}\right\rangle$, simply insert the unity operator in front of it. The summation symbol can then be commuted to the front because $\left\langle\bar{q}, L_{1}\right|$ does not depend on $j$.

$<$ Note to editor, typesetter, etc. Please be careful of the arrangement of Eq. (12). The vertical arrow points directly before $s_{i}$ to indicate the expression below being shoved in at that point. A vertical arrow with a larger arrowhead would be better; I used what was available. Also, it is tremendously important to use the special symbol $\mathbb{1}$. I can supply this symbol as a computer file (encapsulated postscript, eps), or you may already have it on a floppy/CD that I gave you, or you may have this symbol in your resources. Contact me at jworthey@starpower.net or 301-977-3551. JAW.>

Eq. (12) and (13) express the key idea of a matrix to predict color rendering effects. To make use of Eq. (13) may require tristimulus vectors and matrices to be transformed so that they refer to another basis, such as $\{\bar{x}, \bar{y}, \bar{z}\}$. One way to do this is by using the unity operator. Suppose that the pivotal light is $L_{0}$. Let $\langle\bar{x}|$ be post-multiplied by unity operator (D6b):

$$
\langle\bar{x}|=\langle\bar{x}| \mathbb{1}=\sum_{j=1}^{3}\left\langle\bar{x} \mid L_{0} \bar{q}_{j}\right\rangle\left\langle\bar{q}_{j}\right|
$$

and similarly for $\langle\bar{y}|$ and $\langle\bar{z}|$. The result is exact, not approximate, because $\{\langle\bar{x},\langle\bar{y} \overline{\mid},\langle\bar{z}|\}$ are linear combinations of the 3 functions $\left\langle\overline{q_{j}}\right.$.

In matrix notation,

$$
\left[\begin{array}{c}
\langle\bar{x}| \\
\langle\bar{y}| \\
\langle\bar{z}|
\end{array}\right]=\left[\begin{array}{ccc}
\left\langle\bar{x} \mid L_{0} \bar{q}_{1}\right\rangle & \left\langle\bar{x} \mid L_{0} \bar{q}_{2}\right\rangle & \left\langle\bar{x} \mid L_{0} \bar{q}_{3}\right\rangle \\
\left\langle\bar{y} \mid L_{0} \bar{q}_{1}\right\rangle & \left\langle\bar{y} \mid L_{0} \bar{q}_{2}\right\rangle & \left\langle\bar{y} \mid L_{0} \bar{q}_{3}\right\rangle \\
\left\langle\bar{z} \mid L_{0} \bar{q}_{1}\right\rangle & \left\langle\bar{z} \mid L_{0} \bar{q}_{2}\right\rangle & \left\langle\bar{z} \mid L_{0} \bar{q}_{3}\right\rangle
\end{array}\right]\left[\begin{array}{c}
\left\langle\bar{q}_{1}\right| \\
\left\langle\bar{q}_{2}\right| \\
\left\langle\bar{q}_{3}\right|
\end{array}\right] .
$$

The square transformation matrix can be called $\mathbf{T}(\perp \rightarrow \mathrm{XYZ})$. The matrix inverse will give the reverse transformation: $\mathbf{T}(\mathrm{XYZ} \rightarrow \perp)=\mathbf{T}^{-1}(\perp \rightarrow \mathrm{XYZ})$. $\mathbf{T}(\perp \rightarrow \mathrm{XYZ})$ is easy to calculate within a computer language that permits matrix operations. Note that 


$$
\left.\mathbf{T}(\mathrm{XYZ} \rightarrow \perp)=\left[\begin{array}{c}
\langle\bar{x}| \\
\langle\bar{y}| \\
\langle\bar{z}|
\end{array}\right]\left[\left|L_{0} \bar{q}_{1}\right\rangle\left|L_{0} \bar{q}_{2}\right\rangle|| L_{0} \bar{q}_{3}\right\rangle\right] .
$$

With the vectors thus collected into matrices, the transformation matrix can be found by one line of programming. A similar technique may be used with Eq. (14). The matrices in Eq. (D9) are large, with dimensions such as $3 \times 471$ and $471 \times 3$. Now let $|N\rangle$ be any radiance presented to the CIE 1931 observer. Then $X=\langle\bar{x} \mid N\rangle$, etc. Post-multiplying Eq. (D8) by $|N\rangle$, we see that the same square matrix will convert tristimulus values. That is,

$$
\left[\begin{array}{lll}
X & Y & Z
\end{array}\right]^{\prime}=\mathbf{T}(\perp \rightarrow X Y Z)\left[Q_{1} Q_{2} Q_{3}\right]^{\prime} .
$$

Eq. (D10) was derived without reference to a particular light or surface, to emphasize its generality.

Eq. (13) or (15) is the color rendering prediction, expressed in terms of the orthonormal basis. The transformation of Eq. (D8), and its inverse, can convert the color rendering prediction to another basis, such as $\{X, Y, Z\}$. Eq. (15) is first multiplied through on the left by $\mathbf{T}(\perp \rightarrow \mathrm{XYZ})$. Then $Q\left(\perp, L_{0}, s_{i}\right)$ is replaced by $\mathbf{T}^{-1}(\perp \rightarrow \mathrm{XYZ})[X Y Z]^{\prime}$. This transforms the color rendering equation to

$$
\left[\begin{array}{c}
X \\
Y \\
Z
\end{array}\right]_{L 1} \approx \mathbf{T}(\perp \rightarrow \mathrm{XYZ}) \mathbf{P}\left(\perp, L_{0} \rightarrow L_{1}\right) \mathbf{T}^{-1}(\perp \rightarrow \mathrm{XYZ})\left[\begin{array}{c}
X \\
Y \\
Z
\end{array}\right]_{L 0} .
$$

A color rendering matrix in the non-opponent $\mathrm{XYZ}$ system is thus created from one derived in the orthonormal system:

$$
\mathbf{P}\left(\mathrm{XYZ}, L_{0} \rightarrow L_{1}\right)=\mathbf{T}(\perp \rightarrow \mathrm{XYZ}) \mathbf{P}\left(\perp, L_{0} \rightarrow L_{1}\right) \mathbf{T}^{-1}(\perp \rightarrow \mathrm{XYZ}) .
$$

Conceptually, the tristimulus vector on the right in Eq. (D11) is converted to the orthonormal system, the color rendering matrix $\mathbf{P}\left(\perp, L_{0} \rightarrow L_{1}\right)$ is applied, and then the result is converted to XYZ form. Eq. (D9) and (D12) generalize to convert to any other system, such as Guth's original system (Appendix A). The conversion (D12) is easily done for such a use as plotting estimated color shifts in the XYZ system, Figs. 2-4. For interpreting the elements of $\mathbf{P}$, it makes more sense to stick with an opponent system such as the orthonormal one, or Guth's.

\section{Textbook origins}

Most of the mathematical expressions in this article can be read as simple matrix operations. However, the idea of approximating a function by a series, and the steps that call on the orthogonality relation, $\left\langle b_{i} L_{0} \mid b_{j}\right\rangle=\delta_{i j}$, these come from Fourier Series. The theorems of Fourier Series textbooks will carry over, including the proof that each nonzero term added to the series 
reduces the sum-squared error of the approximation. The technique of generalized Fourier series methods leading to matrix elements with specific meanings comes from quantum mechanics. Curiously, the unity operator above, Eq. (D6), is a projection operator like Cohen's Matrix R. Indeed if $L_{0}=1$ (the Equal Energy light), and $k=3$, then $\mathbb{1}=\mathbf{R}$. Here, rather than emphasize the unity operator as a large square matrix, we allow it to fade away in the derivation of matrix $\mathbf{P}$. 


\section{References}

1. James A. Worthey, "Color rendering: asking the question," Color Res. Appl., in press.

2. James A. Worthey, "Opponent-colors approach to color rendering," J. Opt. Soc. Am. 72(1):7482 (1982).

3. Jozef Cohen, "Dependency of the spectral reflectance curves of the Munsell color chips," Psychonom. Sci. 1, 369-370 (1964).

4. Jozef B. Cohen and William E. Kappauf, "Metameric color stimuli, fundamental metamers, and Wyszecki's metameric blacks," Am. J. Psych. 95(4):537-564 (1982).

5. Sherman Lee Guth, Robert W. Massof, and Terry Benzschawel, "Vector model for normal and dichromatic color vision," J. Opt. Soc. Am. 70, 197-212 (1980).

6. William A. Thornton, "Three-color visual response," J. Opt. Soc. Am. 62(3):457-459 (1972). William A. Thornton, "Luminosity and color-rendering capability of white light," J. Opt. Soc. Am. 61(9):1155-1163. William A. Thornton, "A simple picture of matching lights," J. Illum. Eng. Soc. 8(2):78-85 (1979).

7. Ohta, Noboru, "A simplified method for formulating pseudo-object colors," Color Res. Appl. 7, 78-81 (1982).

8. L. Maloney, "Evaluation of linear models of surface spectral reflectance with small numbers of parameters," J. Opt. Soc. Am. A 3, 1673-1683 (1986).

9. D. Marimont, and B. A. Wandell, "Linear models of surface and illuminant spectra," J. Opt. Soc. Am. A 9, 1905-1913 (1992).

10. Michael J. Vrhel, Ron Gershon, and Lawrence S. Iwan, "Measurement and analysis of object reflectance spectra," Color Res. Appl. 19, 4-9, (1994). These data were obtained in digital form by ftp from server ftp.eos.ncsu.edu in directory/pub/spectra.

11. Deane B. Judd, David L. MacAdam, and Günter W. Wyszecki, "Spectral distribution of typical daylight as a function of correlated color temperature," J. Opt. Soc. Am. 54,1031-1040 (1964).

12. Michael H. Brill, “A non-PC look at principal components," Color Res. Appl. 28, 69-73 (2003).

13. CIE 13.3-1995, Method of measuring and specifying colour rendering properties of light sources, 3rd edition. This edition contains the same technical recommendations as the $2 \mathrm{nd}$ edition of 1974, but has been updated with regard to spectroradiometric and calculation practice.

14. Eugen Merzbacher, Quantum Mechanics, Wiley, New York, 1961, pp. 306-308; P. A. M. 
Dirac, The Principles of Quantum Mechanics, 4th ed., Oxford, London, 1958, pp. 18-22.

15. James A. Worthey, "Limitations of color constancy,” J. Opt. Soc. Am. A 2, 1014-1026 (1985).

16. James A. Worthey, "Calculation of metameric reflectances," Color Research and Application 13(2): 76-84 (April 1988).

17. Günter W. Wyszecki and W. S. Stiles, Color Science (Wiley, New York, 1967); fluorescent light spectra on p. 37.

18. Spectral measurements by the author in 1985 .

19. Data from William Thornton in 2000.

20. Data from Nick Lena in 2000.

21. J. B. Maas, J. K. Jayson, and D. A. Kleiber, "Effects of spectral differences in illumination on fatigue," Journal of Applied Psychology 59(4):524-526, 1974.

22. Lumichrome data taken in 2000 from a web page that no longer exists.

23. Data from William Thornton in 1980.

24. Solux data from Philip Bradfield, Tailored Lighting Inc., in 2000.

25. Spectra extracted from graphs in web pages of Philips Lighting Company in 2001, 7 filledtriangle types; see http://www.lighting.philips.com/ .

26. http://www.sulfurlamp.com; spectrum taken from web site in 2002.

27. http://www.gelighting.com/eu/contactus/faq.html ; http://www.ece.uiuc.edu/pubs/centhist/six/illumin.htm .

28. Kevin P. McGuire, personal communication, 1998. Also see the web site of Tailored Lighting Inc., http://www.soluxtli.com/ .

29. Robert M. Boynton, "Minimally distinct borders," in Visual Psychophysics and Physiology, J. C. Armington, J. Krauskopf, and B. R. Wooten, eds. (Academic, New York, 1978).

30. F. S. Frome, S. L. Buck, and R. M. Boynton, "Visibility of borders: separate and combined effects of color differences, luminance contrast, and luminance level," J. Opt. Soc. Am. 71, 145150 (1981).

31. P.B. Kruger, S. Nowbotsing, K.R. Aggarwala, S. Mathews, "Small amounts of chromatic 
aberration influence dynamic accommodation," Optom. Vis. Sci. 72(9):656-66, 1995 September.

32. James A. Worthey, "Effect of veiling reflections on vision of colored objects," Journal of the IES 18(2):10-15 (Summer 1989).

33. James A. Worthey, "Lighting quality and light source size," Journal of the IES 19(2):142148 (Summer 1990)

34. Heschong-Mahone group, "Daylighting in schools: an investigation into the relationship between lighting and human performance," August 20, 1999;

"Skylighting and retail sales: an investigation into the relationship between lighting and human performance," August 20, 1999. Reports available from Heschong-Mahone Group, 11626 Fair Oaks Blvd. \#302, Fair Oaks, CA 95628; or online from http://www.h-m-g.com/ .

35. Peter Signell, quantum mechanics lectures, 1967.

36. Smith, V. C. and Joel Pokorny, "Spectral sensitivity of the foveal cone photopigments between 400 and 500 nm," Vision Res. 15: 161-171 (1975).

37. Andrew Stockman and Lindsay T. Sharpe, Color and Vision database, http://www.cvrl.org/ .

38. Sherman Lee Guth, N. J. Donley and R. T. Marrocco, "On luminance additivity and related topics," Vision Res. 9, 537-575 (1969).

39. H. J. Trussell, "Applications of Set Theoretic Methods to Color Systems," Color Research and Application 16(1):31-41, (February 1991).

40. Th. Keusen, "Multispectral color system with an encoding format compatible with the conventional tristimulus model," Journal of Imaging Science and Technology 40(6):510-515, 1996. 


\section{Color Rendering: a Calculation That Estimates Colorimetric Shifts James A. Worthey \\ Figure Captions}

Figure 1. Set of orthonormalized opponent color functions. As color matching functions, these are equivalent to the usual CIE $2^{\circ}$ observer. Solid $=$ achromatic sensitivity $\overline{q_{1}}(\lambda)$, proportional to the familiar $\bar{y}(\lambda)$. Long dashes $=\overline{q_{2}}(\lambda)=$ red-green sensitivity. Short dashes $=\overline{q_{3}}(\lambda)=$ blueyellow sensitivity.

Figure 2. Chromaticity shifts of 36 Munsell papers are graphed. Arrow tails show the chromaticities under JMW daylight, while arrow heads represent chromaticities under Cool White fluorescent light. Both lights have the same chromaticity, $(0.3825,0.3850)$, indicated by + . Solid arrows are computed exactly, while the dashed arrows are estimated according to Eq. (13).

Figure 3. The lighting transition is the same as in Fig. 2, from JMW daylight to Cool White fluorescent of the same chromaticity, $(0.3825,0.3850)$, indicated by + . Now only 8 Munsell papers are represented, the ones used in the Color Rendering Index method. Again, the solid arrows show exact results, while the dashed arrows are estimated using Eq. (13).

Figure 4. Again the 36 Munsell papers are seen, but the transition is from JMW daylight at $10000 \mathrm{~K}$ to JMW daylight at $4800 \mathrm{~K}$. Solid and dashed arrows are used as before.

Figure 5. Matrix element $P_{22}$, the gain for red and green, is graphed versus correlated color temperature for various lights. The pivotal light is Equal Energy. See text for details.

Figure 6. Similar to Fig. 5, but matrix element $P_{33}$, the gain for blue and yellow, is graphed. See text.

Figure 7. Comparison of idealized prime color to a prime color light that was once commercial, plus two other lights of similar chromaticity. The heavy solid line, seen as 3 narrow triangles, is the idealized prime color light, realistically graphed except that it is scaled by a factor of 0.05 ; $(\mathrm{x}, \mathrm{y})=(0.2695,0.2892)$. The thin solid curve is the prime color fluorescent light, $(0.2695$, $0.2892)$; short dashes $=11,272 \mathrm{~K}$ blackbody, $(0.2746,0.2810)$; long dashes $=$ JMW daylight, $(0.2695,0.2891)$.

Figures are supplied in a separate pdf file. 